\title{
Resistência do solo à penetração em função do sistema de cultivo e teor de água do solo ${ }^{1}$
}

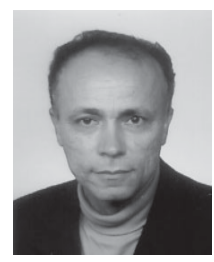

Joaquim O. Pereira², Jair A. C. Siqueira ${ }^{3}$, Miguel A. Uribe-Opazo ${ }^{4}$ \& Suedêmio de L. Silva ${ }^{5}$

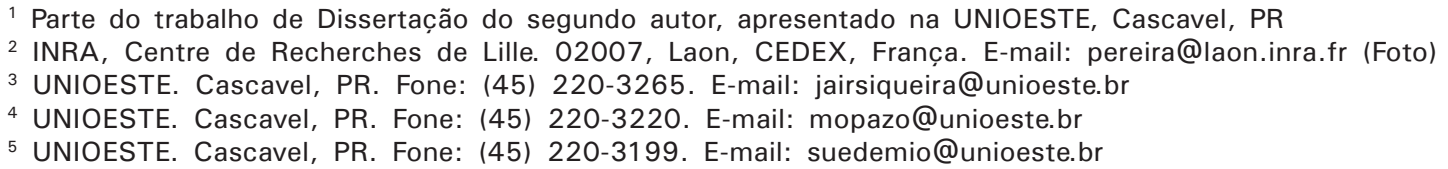

Protocolo $153-14 / 12 / 2000$

\begin{abstract}
Resumo: As variações da resistência do solo à penetração, no cultivo mínimo (escarificação mais semeadura) e plantio direto, foram avaliadas em um Latossolo Roxo distrófico (Oxisol, segundo a Soil Taxonomy) com textura argilosa, com os teores de água do solo de 33, 37 e 39\%, na cultura do milho. Determinou-se a resistência do solo à penetração antes e imediatamente após o preparo do solo e após a colheita, em que os resultados obtidos permitiram concluir-se que tanto os teores de água do solo como os sistemas de cultivo revelaram efeito significativo na resistência do solo à penetração, após o preparo. O teor de água, de 33\%, propiciou redução da resistência do solo à penetração, em ambos os sistemas, com maior redução no sistema de cultivo mínimo e os teores de 37 e $39 \%$ propiciaram aumento da resistência do solo nos dois sistemas de cultivo, com acréscimo maior no sistema de plantio direto.
\end{abstract}

Palavras-chave: resistência do solo à penetração, plantio direto, cultivo mínimo

\section{Soil penetration resistance as a function of tillage system and soil water content}

\begin{abstract}
The effect of tillage systems (minimum-tillage and no-tillage) and water contents (33, 37 and $39 \%$ ) on the soil strength was evaluated on the Oxisol, with the corn yield. The resistance of the soil to penetration was determined before the tillage and immediately after the tillage and after the harvest. The results obtained permit to conclude that the water contents of the soil, as well as the tillage systems affected significantly the resistance of the soil to the penetration after the tillage. The water content of $33 \%$ caused a reduction of the soil penetration resistance, in both systems, with larger reduction in the system of the minimum-tillage. The water content of 37 and $39 \%$ caused an increase of the soil penetration resistance in both the systems with larger increment in the no-tillage system.
\end{abstract}

Key words: soil penetration resistance, no-tillage, minimum-tillage

\section{INTRODUÇÃO}

A resistência do solo à penetração é uma das características que melhor representam as condições de desenvolvimento das raízes das plantas (Tormena \& Roloff, 1996). Segundo Larson \& Allmaras (1971) o desenvolvimento radicular é restringido pela elevada resistência do solo, associada à compactação deste.

Taylor et al. (1966) e Nesmith (1987) determinaram o valor de $2 \mathrm{MPa}$ como resistência crítica à penetração das raízes, em experimento com a cultura de soja e trigo. Por outro lado, mudanças na resistência do solo à penetração, causadas por deslocamento de máquinas agrícolas, dependem do teor de água do solo (Thangavadivelu, 1994).

Hill \& Cruse (1985) desenvolveram um trabalho em duas áreas experimentais, durante dois e oito anos contínuos. A resistência do solo à penetração das raízes foi avaliada com a cultura do milho nos sistemas de cultivo mínimo, plantio direto e preparo convencional. Os autores verificaram que todos os sistemas de cultivo apresentaram efeitos significativos de resistência à penetração na área com oito anos de trabalho. Em ambas as áreas experimentais, os sistemas de cultivo mínimo e plantio direto apresentaram maior resistência do solo à penetração quando comparados com o sistema convencional.

Culley et al. (1987) observaram que no plantio direto o tráfego das máquinas, associado apenas à semeadura e à aplicação de herbicidas, eliminou os benefícios da baixa resistência à penetração e o grau de capacidade de infiltração. Para eles, a resistência do solo à penetração foi um indicador mais sensível dos efeitos do tráfego que a densidade do solo. 
Por sua vez, Hermawan \& Cameron (1993) estudaram o efeito do preparo convencional e do cultivo mínimo sobre a resistência do solo à penetração em um solo argiloso e os resultados mostraram que o sistema de preparo convencional propiciou maior resistência do solo à penetração, quando comparado com os resultados obtidos no cultivo mínimo; já Cassel (1982) constatou que o preparo do solo com escarificador resultou em menor índice de cone nas entrelinhas e na faixa destinada ao tráfego do trator, com relação ao preparo convencional. Sete meses após o preparo nas entrelinhas sem tráfego, a vantagem do preparo com escarificador, ainda persistia na camada de $0-40 \mathrm{~cm}$ de profundidade.

Enfim, face ao exposto avaliou-se, neste trabalho, a resistência do solo à penetração, em um solo argiloso com três teores de água, com a cultura do milho no cultivo mínimo e plantio direto.

\section{MATERIAL E MÉTODOS}

O experimento foi realizado na área do Campo Experimental da COODETEC - Cooperativa Central Agropecuária de Desenvolvimento Tecnológico e Econômico Ltda, em um Latossolo Roxo distrófico (Oxisol, segundo a Soil Taxonomy) com textura argilosa, cujas coordenadas geograficas são: Latitude $24^{\circ} 48^{\prime}$ 'S e Longitude $53^{\circ} 26^{\prime} \mathrm{W}$ com altitude de $760 \mathrm{~m}$. O clima da região é temperado mesotérmico e super-úmido, com precipitação anual em torno de $1920 \mathrm{~mm}$ e temperatura média anual de $21^{\circ} \mathrm{C}$. A área do experimento vinha sendo cultivada há dez anos, com alternância entre as culturas de soja e milho, empregan-do-se o sistema de plantio direto.

O experimento foi realizado de janeiro a junho de 1998, empregando-se dois sistemas de cultivo - plantio direto e cultivo mínimo (escarificação mais semeadura) com a cultura do milho, em área destinada à implantação da cultura, delineada com quatro parcelas contendo seis sub-parcelas com dimensões de 7 × $30 \mathrm{~m}$; entre cada parcela deixou-se uma faixa de bordadura de $0,5 \times 30 \mathrm{~m}$.

A resistência do solo à penetração foi determinada com o uso de um penetrógrafo da marca Soil Control padrão ASAE 313 (ASAE, 1996), em todas as parcelas de 5 - $30 \mathrm{~cm}$ de profundidade, ressaltando-se que as medições foram realizadas antes e imediatamente após o preparo do solo e após a colheita. A compactação do solo foi avaliada em termos de resistência do solo à penetração nos níveis de profundidades de $5,10,15,20$, 25 e $30 \mathrm{~cm}$, nos dois sistemas de cultivo com três teores de água do solo $(33,37$ e 39\%) constituindo um fatorial 2 × 3 × 6 . Os teores de água foram escolhidos em função do teor de água (39\%) de densidade máxima do solo, obtido em laboratório pelo teste de 'Proctor'. Fatores como, época de semeadura, disponibilidade de máquinas agrícolas e frequência de pluviosidade dificultaram a escolha, no campo, de valores de teores de água mais distantes. Por esta razão adotou-se como tratamento um teor de água por dia iniciando pelo de maior valor, utilizando-se tres dias para realização do experimento. Todos os tratamentos foram realizados durante a tarde com os teores de água no campo em tempo real determinados com uso de forno 'Micro onda' e, posteriormente, confirmado pelo teste de estufa com erro de aproximadamente $5 \%$. Os dados foram submetidos a analise de variância fatorial, em nível de 5\% de significância pelo teste "F" e, nos casos de resultados significativos, foram realizadas as comparações de médias entre tratamentos, pelo método de Tukey (Gomes, 1984 e Banzzato \& Kronka, 1992).

\section{RESULTADOS E DISCUSSÃO}

A análise de variância revelou efeito significativo das interações teor de água versus sistema de cultivo e teor de água versus profundidade, da resistência do solo no primeiro período de avaliação.

Os valores médios apresentados na Tabela 1 são apenas reveladores da condição do solo antes do preparo, não remanejado e, nesta condição, mostram que o solo com teor de água de $33 \%$ apresentou maior resistência à penetração quando comparado com os teores de água de 37 ou 39\%, indicando efeito significativo do teor de água na resistência do solo à penetração.

Tabela 1. Valores médios* da resistência do solo à penetração, para os três teores de água do solo e diferentes profundidades, antes da operação de preparo do solo

\begin{tabular}{|c|c|c|c|c|c|c|c|}
\hline \multirow{3}{*}{$\begin{array}{c}\text { Teor de } \\
\text { Água } \\
(\%)\end{array}$} & \multicolumn{6}{|c|}{ Resistência - MPa } & \multirow{3}{*}{$\begin{array}{l}\text { Média } \\
\text { Geral }\end{array}$} \\
\hline & \multicolumn{6}{|c|}{ Profundidade - $\mathrm{cm}$} & \\
\hline & 5 & 10 & 15 & 20 & 25 & 30 & \\
\hline 33 & 0,15 & & & & & & \\
\hline 37 & 0,00 & $89 \mathrm{a}$ & 34 &, 54 &, 56 & $59 \mathrm{a}$ & $1,35 \mathrm{~B}$ \\
\hline 39 & 0,00 &, $52 \mathrm{a}$ & 412 & $25 \mathrm{a}$ & $40 \mathrm{a}$ & $71 \mathrm{a}$ & $1,22 \mathrm{~B}$ \\
\hline
\end{tabular}

* Letras maiúsculas ou minúsculas iguais entre colunas e linhas, respectivamente, não diferem estatisticamente $(\mathrm{p}<0,05)$ pelo teste de Tukey

Os valores indicam ainda que, embora não tenha ocorrido diferença estatística pelo teste de Tukey em nível de 5\% de probabilidade da resistência do solo à penetração, nas profundidades de 10 a $30 \mathrm{~cm}$ com cada teor de água, pode-se visualizar aumento da resistência, na profundidade de 10 e $15 \mathrm{~cm}$, comportamento que se verifica em todos os teores de água. Os resultados podem estar associados à intensivas práticas de cultivo, continuamente realizadas nesta área, de maneira inadequada, inclusive o plantio direto, com o teor de água e a pressão de contato pneu-solo, na época das operações de cultivo, excessivamente altos, causando na camada trabalhada, um solo estruturalmente deficiente, tendo provavelmente, concorrido para isto, os efeitos da interação do teor de água versus energia de compactação do solo versus estado estrutural inicial da camada do solo trabalhada (Papy, 1987; Richard et al., 1999; Abu-Hamdeh, 2000 e Yavuzcan, 2000).

Os valores de teor de água das Tabelas 2 e 4 referem-se aos obtidos no período de instalação dos tratamentos e servem como indicadores do comportamento da evolução do sistema de cultivo, uma vez que, após a colheita, a resistência do solo foi obtida a um mesmo teor de água.

Os resultados da Tabela 2, embora não apresentem valores estatisticamente diferentes, evidenciam crescimento da resistência do solo, de 1,23 para 1,51 $\mathrm{MPa}$ do teor de água 33 para $37 \%$ no sistema de cultivo mínimo, e de 1,79 para $1,92 \mathrm{MPa}$ no sistema de plantio direto, concordando com Culley et al.

Tabela 2. Valores médios da resistência do solo à penetração*, para os sistemas de cultivo mínimo (CM) e plantio direto (PD) em três teores de água do solo, após a colheita de milho

\begin{tabular}{cccc}
\hline \multirow{2}{*}{$\begin{array}{c}\text { Sistema de } \\
\text { Cultivo }\end{array}$} & \multicolumn{3}{c}{ Resistência - MPa } \\
\cline { 2 - 4 } & 33 & 37 & 39 \\
\cline { 2 - 4 } CM & $1,23 \mathrm{aA}$ & $1,51 \mathrm{aA}$ & $1,39 \mathrm{aA}$ \\
$\mathrm{PD}$ & $1,79 \mathrm{aB}$ & $1,92 \mathrm{aB}$ & $1,47 \mathrm{bA}$ \\
\hline * Letras maiúsculas ou minúsculas iguais entre colunas e linhas, respectivamente, não diferem
\end{tabular}
estatisticamente $(\mathrm{p}<0,05)$ pelo teste de Tukey 
(1987). Os resultados revelam que o uso de máquinas agrícolas em solo argiloso com teor de água na faixa ótima de "Proctor", pode conduzir a um aumento da compactação do solo, o que fica mais caracterizado quando se observa a diminuição da resistência do solo de 0,12 cultivo mínimo (CM) e $0,45 \mathrm{MPa}$ plantio direto (PD) trabalhando com o teor de água de 37 e 39\%. Os resultados ainda são mais evidentes com o sistema de plantio direto em que a mobilização do solo é mínima. O fato dos valores dos teores de água nesta faixa tornarem-se muito próximos, pode ter favorecido a obtenção de resultados não significativos.

Os valores das médias apresentadas na Tabela 3, revelam aumento da resistência do solo à penetração, em ambos os sistemas de cultivo e em todas as profundidades do solo, com incremento maior no sistema de PD que apresentou, na profundidade de 10 a $30 \mathrm{~cm}$ ocorrência de valores médios de resistência do solo a penetração, variando de 1,98 a 2,19 MPa. Valores similares foram encontrados por Taylor et al. (1966) e Nesmith (1987) que determinaram o valor de $2 \mathrm{MPa}$ como resistência crítica à penetração das raízes. Verifica-se também, na Tabela 3, que o sistema de cultivo mínimo foi o fator que contribuiu significativamente na diminuição da resistência do solo à penetração na camada de $10 \mathrm{a} 20 \mathrm{~cm}$, o que se atribui a ação modificadora pelo escarificador da estrutura inicial do solo.

Tabela 3. Valores médios da resistência do solo à penetração, $(\mathrm{MPa})$, entre os sistemas de cultivo mínimo (CM) e plantio direto (PD) em diferentes profundidades, após a colheita de milho

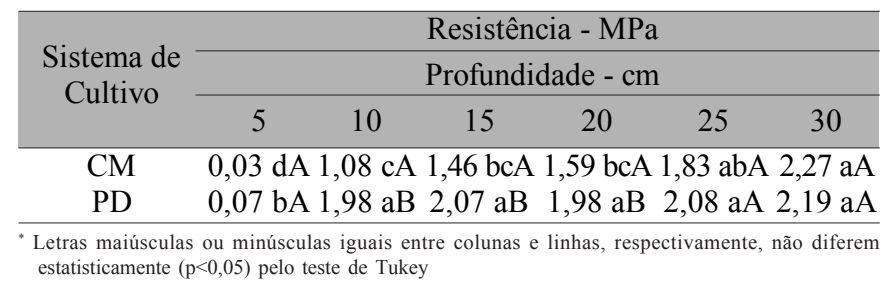

Os resultados da Tabela 4 revelam aumento significativo de resistência do solo para os teores de água de $33 \%$ e $37 \%$ da profundidade de 5 para $10 \mathrm{~cm}$ e para o teor de $39 \%$ da profundidade de 5 para $10 \mathrm{~cm}$, de 10 para $15 \mathrm{~cm}$ e de 20 para $25 \mathrm{~cm}$. As profundidades de 10 a $30 \mathrm{~cm}$ com o teor de água de $33 \%$ apresen- taram pouca variação na resistência do solo, com valores de 1,72 a $1,84 \mathrm{MPa}$, respectivamente, não revelando diferença estatística significativa entre as profundidades; entretanto, pode-se verificar tendência forte de compactação do solo na camada de 5 a $10 \mathrm{~cm}$ em todos os teores de água e atribuir este aumento à prática de manejo repetida que se vem realizando nesta área, com alto teor de água do solo. Verificando-se nas Tabelas 3 e 4, a camada de 25 a $30 \mathrm{~cm}$, pode-se perceber que os dados conduzem à ocorrência do efeito cumulativo dos sistemas de cultivo dos anos anteriores, privilegiando o papel do teor de água na mudança do estado estrutural do solo no momento da operação, concordando com Coulomb etal. (1990).

Tabela 4. Comparação de médias da resistência do solo à penetração*, teores de água em diferentes profundidades, após a colheita de milho, nos sistemas de cultivo

\begin{tabular}{ccccccc}
\hline \multirow{2}{*}{$\begin{array}{c}\text { Teor de } \\
\text { Água (\%) }\end{array}$} & \multicolumn{5}{c}{ Resistência - MPa } \\
\cline { 2 - 6 } & 5 & 10 & 15 & 20 & 25 & 30 \\
\hline 33 & $0,04 \mathrm{bA}$ & $1,72 \mathrm{aA}$ & $1,89 \mathrm{aA}$ & $1,76 \mathrm{aA}$ & $1,82 \mathrm{aA}$ & $1,84 \mathrm{aB}$ \\
37 & $0,00 \mathrm{bA}$ & $1,88 \mathrm{aA}$ & $1,96 \mathrm{aA}$ & $2,02 \mathrm{aA}$ & $2,11 \mathrm{aA}$ & $2,30 \mathrm{aAB}$ \\
39 & $0,12 \mathrm{dA}$ & $0,99 \mathrm{cB}$ & $1,45 \mathrm{bcA}$ & $1,58 \mathrm{bcA}$ & $1,91 \mathrm{abA}$ & $2,55 \mathrm{aA}$ \\
\hline "Letras maiúsculas ou minúsculas iguais entre colunas e linhas, respectivamente, não diferem
\end{tabular}
estatisticamente $(\mathrm{p}<0,05)$ pelo teste de Tukey

A Figura 1 mostra a resistência do solo à penetração, em função da profundidade do solo para os dois sistemas de cultivo e três teores de água nos três períodos de observação.

Dos teores de água empregados, apenas o de 33\%, no sistema de cultivo mínimo, revelou efeito maior na diminuição da resistência do solo durante o ciclo da cultura, concluindo-se que uma correlação importante pode estar associada a este fenômeno, como ocorrência de maior área mobilizada, sem provocar danos à estrutura do solo, facilitando o desenvolvimento do sistema radicular das plantas, o que pode ter contribuído para melhorar a estrutura do solo neste tratamento; outrossim, apesar das desvantagens dos teores de água de 37 e 39\% nos dois sistemas de cultivo, observa-se efeito marcante do escarificador imediatamente após o preparo do solo, reduzindo a resistência do solo, até a camada de $20 \mathrm{~cm}$, numa proporção inversa com profundidade do solo evidenciando-se tendência de pequeno aumento da resistência do solo à penetração, após a camada dos $20 \mathrm{~cm}$.
A.

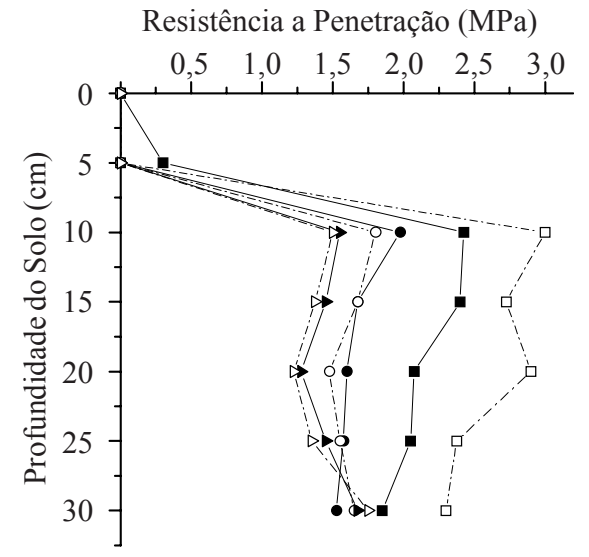

B. Resistência a Penetração (MPa)

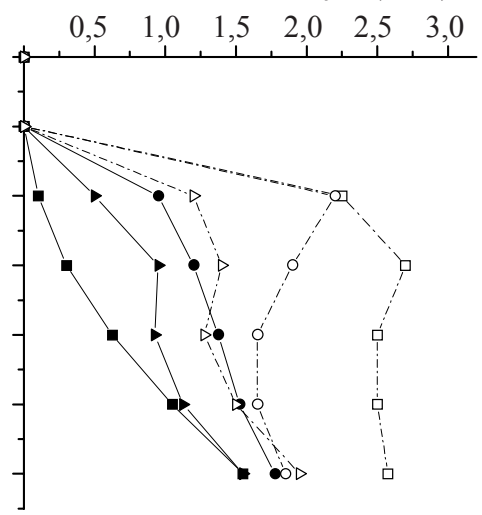

C.

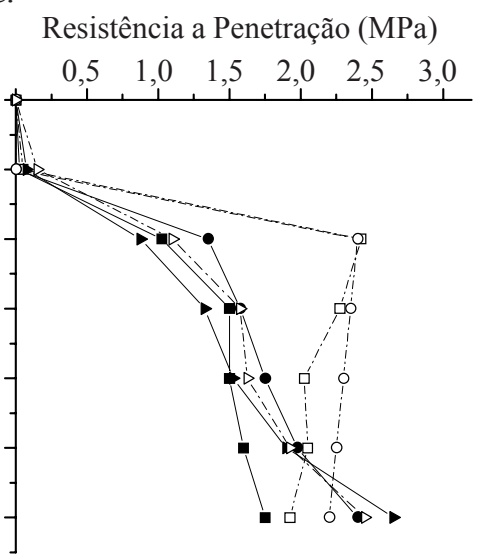

Figura 1. Variação da resistência do solo à penetração nos teores de água do solo de 33, 37 e 39\% nos sistemas de cultivo mínimo (CM) e plantio direto (PD) durante os três períodos de observação: (A) antes do preparo do solo; (B) imediatamente após o preparo do solo; (C) após a colheita do milho 
O sistema de plantio direto apresenta comportamento semelhante ao da resistência do solo, nos três períodos de observação, porém o teor de água, de 39\%, foi o que propiciou aumento considerável após a colheita. Considerando-se que após a passagem da semeadora há tendência na compactação do solo em razão da carga aplicada sobre a área e a inexistência de mobilização do solo, já se esperava um acréscimo na resistência do solo após a semeadura e redução até o período da colheita, em função da atuação do sistema radicular das plantas durante o seu crescimento.

Entre os teores de água avaliados, o teor de $39 \%$ mostrou grande similaridade nos dois sistemas de cultivo durante os três períodos de observação, indicando claramente aumento marcante na resistência do solo. Evidencia-se, ainda, que em todos os períodos de observação o sistema de plantio direto acarretou maior compactação do solo.

\section{CONCLUSÕES}

Os resultados permitiram concluir-se que:

1. O teor de água de $33 \%$ propiciou redução da resistência do solo à penetração, em ambos os sistemas, com diminuição maior no sistema de cultivo mínimo.

2. Os teores de água, de 37 e $39 \%$, propiciaram aumento da resistência do solo nos dois sistemas de cultivo com acréscimo maior no sistema de plantio direto, não sendo observadas diferenças significativas entre eles.

3. O sistema de plantio direto propiciou maior compactação do solo.

4. A compactação do solo está relacionada ao controle do nível de energia, do teor de água e do estado estrutural do solo no momento da operação. Sem o controle destes fatores, qualquer sistema de cultivo induz à compactação do solo.

\section{LITERATURA CITADA}

Abu-Hamdeh, N.H.; Al-Widyan, M.I. Effect of axle load, tire inflation pressure, and tillage system on soil physical properties and crop yield of a Jordanian soil. Transactions of the ASAE, St Joseph, v.43, n.1, p.13-21, 2000.

ASAE - American Society of Agricultural Engineers. Soil cone penetrometer. In: ASAE standards 1996: Standards engineering practices data. St Joseph, 1996. p.719. ASAE S313.2 DEC94

Banzzato, D.A.; Kronka, S.N. Experimentação agrícola, Jaboticabal: FUNEP. 1992. 247p.
Cassel, D.K. Tillage effects on soil bulk density and mechanical impedance. In: Unger, P.W.; van Doren Jr., D.M. (ed.): Predicting tillage effects on soil physical properties and processes. Madison: American Society of Agronomy/ Soil Science Society of America, 1982. p.45-68.

Coulomb, I.; Manichon, H.; Roger-Estrade, J. Evolution de l'état structural sous l'action des systèmes de culture. In : Boiffin, J. ; Marin-Lafleche, A. (ed.) La structure du sol et son évolution: conséquences agronomiques, maîtrise par l'agriculteur. Les colloques de l'INRA, Paris, v.53, p.137-155, 1990.

Culley, J.L.B.; Larson, W.E; Randall, G.W. Physical properties of a typic Haplaquoll under conventional and no-tillage. Soil Science Society of America Journal, Madison, v.51, p.1583-1587, 1987.

Gomes, F.P. A estatística moderna na pesquisa agropecuária. Piracicaba: POTAFOS. 1984. 160p.

Hermawan, B.; Cameron, K.C. Structural changes in a silt loam under long-term conventional or minimum tillage. Soil \& Tillage Research, Amsterdam, v.26, p.139-50, 1993.

Hill, R.Lo.; Cruse, R.M. Tillage effects on bulk density and soil strength of two Mollisoils. Soil Science Society of America Journal, Madison, v.49, p.1270-1273, 1985.

Larson, W.E.; Allmaras, R R. Management factors and natural forces as related compaction. In: Compaction of agricultural soils. American Society of Agricultural Engineers, St. Joseph, p.367-427. 1971.

Nesmith, D.S. Soil compation in double cropped wheat and soybean on Ultissol. Soil Science Society of America Journal, Madison, v.51, p.183-186, 1987.

Papy. F. Comportement d'une couche labourée sous des actions de compactage en fonction de son état hydrique et structural. Agronomie, Paris, v.7, n.2, p.111-121, 1987.

Richard, G.; Boizard, H.; Roger-Estrade, J.; Boiffin, J.; Guerif, J., Field study of soil compaction due to traffic in Northern France: Pore space and morphological analysis of the compacted zones. Soil \& Tillage Research, Amsterdam, v.51,p.151-160, 1999.

Taylor, H.M.; Robertson, G.M.; Parker, J.J. Soil strength root penetrations for medium to coarse textured soil materials. Soil Science, Baltimore, v.102, p.18-22, 1966.

Thangavadivelu, S. Soil response to track and wheel tractor traffic. Journal of Terramechanics. Hannover, v.31, n.1, p.41-50, 1994.

Tormena, C.A.; Roloff, G. Dinâmica da resistência à penetração de um solo sob plantio direto. Revista Brasileira de Ciência do Solo, Campinas, v.20, p.333-339, 1996.

Yavuzcan, H.G. Wheel traffic impact on soil conditions as influenced by tillage system in Central Anatolia. Soil \& Tillage Research, Amsterdam, v.54, 129-138, 2000. 